\title{
Chronic administration of membrane sealant prevents severe cardiac injury and ventricular dilatation in dystrophic dogs
}

\author{
DeWayne Townsend, ${ }^{1}$ Immanuel Turner, ${ }^{1}$ Soichiro Yasuda, ${ }^{1}$ Joshua Martindale, ${ }^{1}$ \\ Jennifer Davis, ${ }^{1}$ Michael Shillingford, ${ }^{1}$ Joe N. Kornegay, ${ }^{2}$ and Joseph M. Metzger ${ }^{1}$ \\ ${ }^{1}$ Department of Integrative Biology and Physiology, University of Minnesota, Minneapolis. \\ 2Department of Pathology and Laboratory Medicine, University of North Carolina, Chapel Hill.
}

\begin{abstract}
Duchenne muscular dystrophy (DMD) is a fatal disease of striated muscle deterioration caused by lack of the cytoskeletal protein dystrophin. Dystrophin deficiency causes muscle membrane instability, skeletal muscle wasting, cardiomyopathy, and heart failure. Advances in palliative respiratory care have increased the incidence of heart disease in DMD patients, for which there is no cure or effective therapy. Here we have shown that chronic infusion of membrane-sealing poloxamer to severely affected dystrophic dogs reduced myocardial fibrosis, blocked increased serum cardiac troponin I (cTnI) and brain type natriuretic peptide (BNP), and fully prevented left-ventricular remodeling. Mechanistically, we observed a markedly greater primary defect of reduced cell compliance in dystrophic canine myocytes than in the mildly affected $m d x$ mouse myocytes, and this was associated with a lack of utrophin upregulation in the dystrophic canine cardiac myocytes. Interestingly, after chronic poloxamer treatment, the poor compliance of isolated canine myocytes remained evident, but this could be restored to normal upon direct application of poloxamer. Collectively, these findings indicate that dystrophin and utrophin are critical to membrane stability-dependent cardiac myocyte mechanical compliance and that poloxamer confers a highly effective membrane-stabilizing chemical surrogate in dystrophin/utrophin deficiency. We propose that membrane sealant therapy is a potential treatment modality for DMD heart disease and possibly other disorders with membrane defect etiologies.
\end{abstract}

\section{Introduction}

Duchenne muscular dystrophy (DMD) is a devastating progressive disease of striated muscle deterioration that results from the loss of the protein dystrophin $(1,2)$. Most patients with DMD develop clinically significant cardiomyopathy during the second decade of life, with heart failure emerging as a leading cause of death in DMD (3-5). Currently there are no effective therapies for the cardiomyopathy of DMD. Numerous therapeutic strategies have been proposed to impact striated muscle disease progression in DMD (6-12). Most of these approaches focus primarily on the skeletal muscle manifestations of the disease, often leaving the deteriorating heart untreated $(6,7,9-11)$. Thus, there is an urgent need to pursue new strategies to prevent, halt, or reverse the emerging heart disease in DMD.

The availability of small and large animal models of DMD has been instrumental in gaining insights into the molecular mechanisms of striated muscle disease progression. The golden retriever muscular dystrophy (GRMD) animal model of DMD has been invaluable for testing leading muscular dystrophy treatment modalities $(9,10,13-22)$. Muscular dystrophy in GRMD animals closely recapitulates the timing and severity of disease progression observed in DMD human patients (23-26). GRMD arose from a spontaneous mutation in intron 6 of the dystrophin locus that eliminates a splice acceptor site, resulting in the skipping of exon 7 and a subsequent frameshift and premature trun-

Conflict of interest: J.M. Metzger is on the scientific advisory board of and holds shares in Phrixus Pharmaceuticals Inc., a company developing novel therapeutics for heart failure.

Citation for this article: J Clin Invest. 2010;120(4):1140-1150. doi:10.1172/JCI41329. cation of the dystrophin protein (27). In addition to the severe skeletal muscle pathology, GRMD animals have prominent cardiac lesions present as early as 6 months of age (25), with ECG abnormalities present at 1 year (28) and profound myocardial contractile abnormalities by 20 months (29).

In comparison to the muscle pathology present in dystrophic humans and dogs, the dystrophic mouse is comparatively mildly affected. Nonetheless, the $m d x$ mouse model of DMD has been widely used and has been absolutely essential for drug, cell, and gene-based therapy investigations. In fact, an understanding of the mechanism(s) underlying the more mild disease in the mouse has provided unique insights into therapeutic approaches to attenuating the disease progression in larger mammals and human patients with DMD. In the dystrophin-deficient mouse heart, the primary defect reported at the level of isolated cardiac myocyte is a reduced compliance to passive extension (30). Reduced myocyte compliance was found to be secondary to calcium influx through apparent micro-tears in the cardiac membrane during passive cell distension (30). The poor passive compliance of cardiac myocytes is physiologically relevant in that during passive filling of the ventricle, individual myocytes are passively extended. The magnitude of these passive extensions is greater with the heightened venous return during periods of stress (30).

Chemical-based membrane sealants have been tested acutely in $m d x$ mice and recently proposed as a new therapeutic approach for cardiac membrane stabilization in muscular dystrophy (30). This treatment modality aims to directly seal the membrane tears that occur in the absence of dystrophin. We recently showed that application of the membrane sealant poloxamer 188 (P188) confers acute cardiac protection in the $m d x$ mouse model of DMD 
A

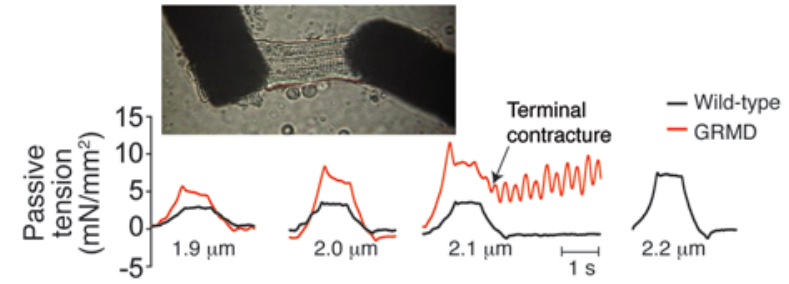

B

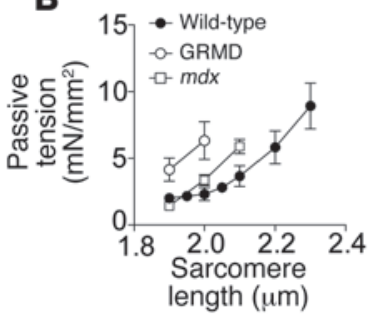

D
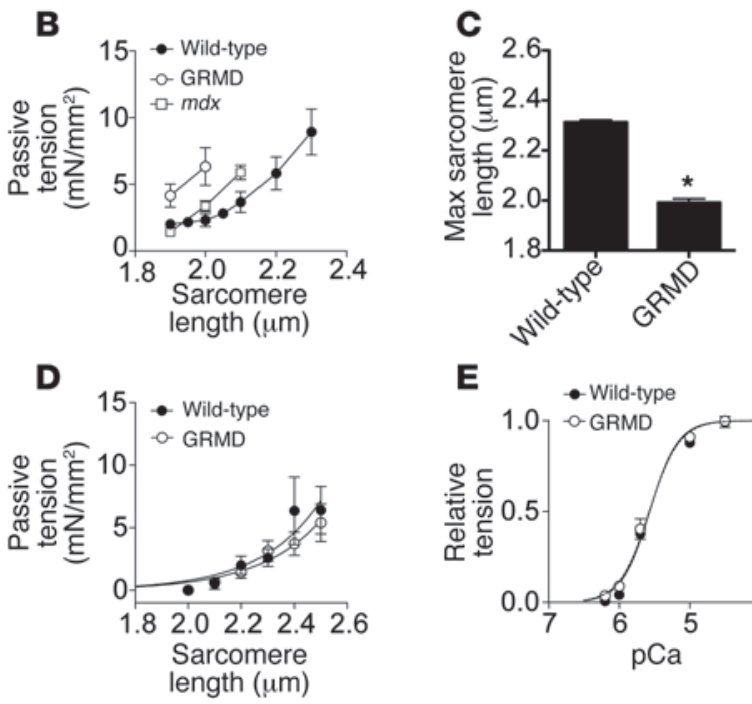

$\mathbf{E}$

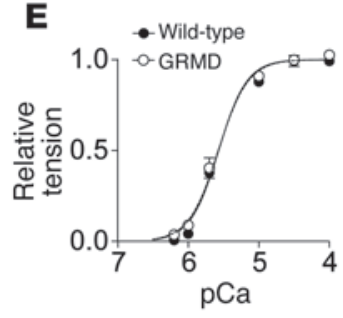

Figure 1

Mechanical properties of adult cardiac myocytes isolated from GRMD dogs. Membrane-intact adult cardiac myocytes were isolated by enzymatic digestion from the hearts of 2 untreated 1-year-old GRMD dogs. (A) Microcarbon fibers were attached to the isolated myocytes, allowing mechanical manipulations and tension measurements (photo). Passive tension tracings from membrane-intact cardiac myocytes extended to sarcomere lengths indicated below the tracing. $\mathrm{mN}$, millinewton. (B) Summary passive extension-tension curves are shown for membrane-intact cardiac myocytes isolated from wild-type and GRMD dogs and $m d x$ mice. (C) Summary of the maximal sarcomere length tolerated by the myocytes. ${ }^{*} P<0.05$. (D) Solubilization of membranes by detergents permits direct assessment of myofilaments. In a solution of nominal calcium, passive extension of myofilaments from wild-type and GRMD myocytes revealed no differences (7-8 cells from 2 dogs for each genotype). (E) Assessment of myofilament calcium sensitivity in membrane-solubilized myocytes revealed no differences between GRMD and wild-type myocytes. Data are from 5-7 myocytes isolated from $2-3$ animals. $\mathrm{pCa},-\log \left[\mathrm{Ca}^{2+}\right]$.

To determine the passive properties of GRMD myocytes, we isolated membrane-intact cardiac myocytes and subjected them to passive extension-tension studies using a unique micro-carbon fiber assay (30). Cardiac myocytes isolated from wild-type dogs well tolerated passive extensions throughout the physiological range of sarcomere lengths (1.8-2.2 $\mu \mathrm{m}$; ref. 31). In marked contrast to wild-type myocytes, GRMD myocytes were highly sensitive to passive extension and showed blunted compliance (Figure 1, $\mathrm{A}-\mathrm{C}$ ). These effects in GRMD myocytes were more pronounced than in the $m d x$ myocyte (Figure 1B; ref. 30). The marked sensitivity to passive extension could result from perturbations of the sarcomeric myofilaments. To directly assess this possibility, we chemically removed the surface membrane. This solubilization of the membrane allows direct investigation of the properties of the underlying myofilament-based tension-extension properties of the cell. Examination of GRMD dystrophic myofilaments revealed passive properties and calcium sensitivity similar to those of wildtype canine myocytes (Figure 1, D and E). These data indicate that the poor compliance of dystrophic myocytes stems from abnormalities requiring an intact sarcolemmal membrane.

Baseline in vivo bemodynamics in GRMD animals. In order to address the importance of poor myocyte compliance on global hemodynamic function of the heart, we subjected GRMD dogs to invasive catheter-based hemodynamic assessment. Eight adult GRMD dogs and 10 wild-type dogs were utilized in these studies (Table 1). Animals were anesthetized, and baseline blood samples were drawn. Serum levels of cardiac troponin I (cTnI) were significantly elevated in GRMD dogs immediately after induction of anesthesia compared with unaffected anesthetized wild-type dogs (Figure 2A). At rest, GRMD animals had normal serum cTnI levels (see below), indicating that general anesthesia alone provokes significant cardiac damage in GRMD but not in normal dogs. Anesthesia had no significant effect on elevated serum levels of creatine kinase (CK) (Figure $2 \mathrm{~B}$ and below). In contrast to the $m d x$ mouse model of DMD (30), dystrophic dog hearts had no significant reduction in end-diastolic volume (Figure 3), nor was end-diastolic pressure elevated (Table 2).

GRMD dogs had significant hypertension, with increased ventricular and femoral artery systolic pressures, compared with wildtype dogs (Table 2). Arterial elastance and total peripheral resistance were increased (Table 2), suggesting differences within the 


\section{Table 1}

Initial ages, weights, selected baseline hemodynamic parameters, and randomized treatment groups in GRMD and wild-type dogs

\begin{tabular}{|c|c|c|c|c|c|c|}
\hline Animal no. & Genotype & Age (mo) & Weight (kg) & Treatment group & $\begin{array}{l}\text { LV end-diastolic } \\
\text { vol (ml) }\end{array}$ & $\begin{array}{c}\text { LV systolic } \\
\text { pressure }(\mathrm{mmHg})\end{array}$ \\
\hline 1 & GRMD & 12.0 & 12.5 & Saline & 18.7 & 94.7 \\
\hline 2 & GRMD & 17.0 & 13.4 & Saline & 56.7 & 114.6 \\
\hline 3 & GRMD & 13.8 & 11.2 & P188 & 23.7 & 88.5 \\
\hline 4 & GRMD & 17.3 & 13.2 & P188 & 46.0 & 130.4 \\
\hline 5 & GRMD & 16.2 & 14.2 & P188 & 29.9 & 123.8 \\
\hline 6 & GRMD & 17.9 & 17.1 & Saline & 28.7 & 121.1 \\
\hline 7 & GRMD & 13.2 & 13.1 & P188 & 24.9 & 114.3 \\
\hline 8 & GRMD & 13.8 & 12.7 & Saline & $\mathrm{N} / \mathrm{A}$ & 95.7 \\
\hline 9 & WT & $\approx 12$ & 18.7 & $\mathrm{~N} / \mathrm{A}$ & 52.2 & 84.1 \\
\hline 10 & WT & $\approx 12$ & 17.3 & $\mathrm{~N} / \mathrm{A}$ & 46.0 & 88.6 \\
\hline 11 & WT & $\approx 12$ & 18.4 & $\mathrm{~N} / \mathrm{A}$ & 37.8 & 90.8 \\
\hline 12 & WT & $\approx 12$ & 17.7 & $\mathrm{~N} / \mathrm{A}$ & 39.2 & 90.4 \\
\hline 13 & WT & $\approx 12$ & 19.9 & $\mathrm{~N} / \mathrm{A}$ & 29.0 & 87.6 \\
\hline 14 & WT & $\approx 12$ & 19.5 & $\mathrm{~N} / \mathrm{A}$ & 36.1 & 98.2 \\
\hline 15 & WT & $\approx 12$ & 17.9 & $\mathrm{~N} / \mathrm{A}$ & 46.0 & 87.6 \\
\hline 16 & WT & $\approx 12$ & 18.9 & $\mathrm{~N} / \mathrm{A}$ & 34.1 & 85.5 \\
\hline 17 & WT & $\approx 12$ & 17.6 & $\mathrm{~N} / \mathrm{A}$ & 38.8 & 77.4 \\
\hline 18 & WT & $\approx 12$ & 19.4 & $\mathrm{~N} / \mathrm{A}$ & 50.9 & 78.9 \\
\hline
\end{tabular}

Ages of wild-type dogs are approximate. N/A, not applicable.

systemic vasculature of the dystrophic dogs. Differences in arterial function may also explain the increase in maximal rate of relaxation $\left(\mathrm{dP} / \mathrm{dt}_{\mathrm{min}}\right)$ in the absence of other indicators of improved diastolic function (tau and $90 \%$ relaxation time; Table 2 ). Early relaxation of ventricular pressure is aided by the filling of the coronary vasculature, and poor arterial compliance increases the reflection of the systolic pressure pulse back toward the heart. This increase in the reflected pressure pulse increases the pressure in the coronary vasculature and in turn increases the rate of early ventricular relaxation (32).

After baseline measurements, dobutamine was infused $(15 \mu \mathrm{g} / \mathrm{kg} /$ min) until a peak effect was observed, at which time dobutamine infusion was stopped. In response to dobutamine, dystrophic dogs showed evidence of significant cardiac reserve function, with increased systolic and diastolic parameters similar to those observed in wild-type dogs (Figure 3 and Table 2). After dobutamine infusion, a dynamic change in ventricular volume was observed in wild-type dogs; however, these changes were significantly attenuated in dystrophic dogs (Figure 3, E and F).

Acute infusion of membrane sealants in GRMD animals. During the initial hemodynamic protocol, an acute dose of P188 $(460 \mathrm{mg} / \mathrm{kg})$ was administered i.v. over a period of 15 minutes in a total volume of $3 \mathrm{ml} / \mathrm{kg}$. This dose was sufficient to normalize the end-diastolic volume of the $m d x$ mouse (30). In the GRMD heart, this dose of P188 had no significant acute effect on the end-diastolic volume (Figure 4A). No significant changes in enddiastolic volume were observed in wild-type animals infused with equivalent doses of P188. Histological examination of heart sections from 1-year-old GRMD dogs not involved in the chronic study revealed the presence of extensive fibrosis when stained with Sirius red. This fibrosis takes 2 forms: large lesions associated with diseased myocardium and increased levels of fibrosis surrounding relatively normal myocardium (Figure 4B). Analysis of wild-type sections revealed only small amounts of collagen between layers of myocardium (Figure 4C).
We addressed the potential acute effects of P188 on the vasculature by determining several measures of vascular function. The elastic property of the large arteries was not altered by the acute administration of P188 (Supplemental Figure 1; supplemental material available online with this article; doi:10.1172/ JCI41329DS1). Similarly, total peripheral resistance and peripheral blood pressure were unaffected by P188 (Supplemental Figure 1). These data are consistent with the selective action of P188 on the membranes of dystrophic cardiac myocytes.

During the hemodynamic assay, arrhythmic events were infrequent and not different between wild-type and GRMD dogs. Dystrophic dogs had significantly increased baseline heart rate and shortened $\mathrm{P}-\mathrm{R}$ interval compared with wild-type dogs, neither of which were different after administration of dobutamine (Table 2). The ratio of Q-wave to R-wave amplitude was significantly elevated in dystrophic dogs, but this effect was not altered by acute dobutamine (Table 2). Acute infusion of P188 had no significant effects on ECG measurements in wild-type or dystrophic dogs (Supplemental Figure 2).

Chronic infusion of poloxamer in GRMD animals. Continuous intravascular infusion of P188 or saline vehicle control was initiated in adult GRMD animals. Animals were randomized to treatment group. Baseline hemodynamic function was similar in the groups prior to chronic infusion of P188 or saline (Table 1). After the initial hemodynamic assessment, a vascular access port was implanted on the flank and connected to an indwelling jugular catheter. The carotid artery was repaired, all incisions were closed, and the animal was allowed to recover from anesthesia. The next day, an infusion of $0.9 \%$ saline $(0.4 \mathrm{ml} / \mathrm{kg} / \mathrm{h})$ was initiated in both groups, lasting 1 week. The serum half-life of P188 is 18 hours in dogs (33); thus, 1 week is sufficient to wash out any P188 remaining from the acute administration. After this washout and a surgery recovery period, the saline group continued receiving $0.9 \% \mathrm{NaCl}$ at a rate of $0.4 \mathrm{ml} / \mathrm{kg} / \mathrm{h}$, and the $\mathrm{P} 188$ treatment group began receiving $60 \mathrm{mg} / \mathrm{kg} / \mathrm{h} \mathrm{P} 188$ in $0.9 \% \mathrm{NaCl}$ at the same rate $(0.4 \mathrm{ml} / \mathrm{kg} / \mathrm{h})$ for 8 weeks total treatment (Figure $5 \mathrm{~A}$ ). 
A
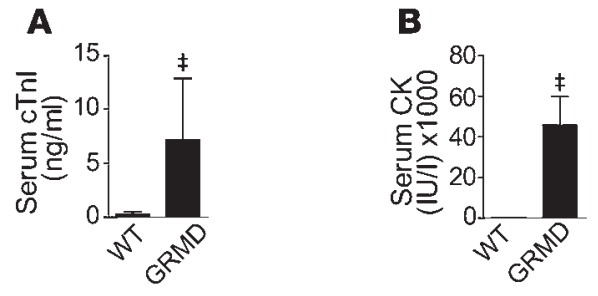

Figure 2

Elevations in serum cTnl after induction of anesthesia in GRMD animals. Serum samples collected within minutes of induction of general anesthesia reveal significant elevations in a biomarker of cardiac injury (A). (B) Significant elevations in serum CK in GRMD dogs. Values are shown as mean \pm SEM; $n=8-10$. ${ }^{\ddagger} P<0.05$ by $t$ test.

During the 8-week chronic infusion period, blood samples were taken periodically to monitor cardiac injury biomarkers. Salinetreated GRMD animals had significantly elevated serum concentrations of cTnI, evidence of cardiac injury. In contrast, serum cTnI levels were completely normal in the P188-treated GRMD animals (Figure 5B; $P<0.05$ vs. saline). Serum CK levels were unchanged by $\mathrm{P} 188$ infusion (Figure 5C). The safety of P188 for both the renal and hepatic systems was assessed. Figure 5, D and E, shows that during the course of the 8-week infusion, the dogs receiving P188 had no change in liver enzymes or evidence of azotemia. These results indicate that administration of P188 at $60 \mathrm{mg} / \mathrm{kg} / \mathrm{h}$ for 8 weeks does not result in kidney or liver damage in GRMD animals.

Myocardial fibrosis is a consistent finding in both DMD patients and GRMD dogs (Figure 4 and refs. 5, 25, 34, 35). Fibrotic lesions are thought to result from myocyte necrosis and subsequent inflammatory response. These fibrotic lesions likely increase the mechanical strain on neighboring myocytes, causing further myocyte necrosis and subsequent fibrosis over time. To assess the ability of chronically administered P188 to limit fibrosis, we stained cardiac tissue with Sirius red to identify mature fibrotic lesions. Upon quantification of the fibrotic area, GRMD animals receiving P188 had significantly less $(P<0.05)$ fibrosis than the GRMD group receiving saline (Figure $6, \mathrm{~A}$ and $\mathrm{B}$ ). While P188 blunted lesion formation in GRMD animals, it did not restore myocardial lesion content to wild-type levels (Figure 6B). Chronic P188 administration to adult GRMD animals did fully block increases in the heart failure biomarker BNP (Figure 6C; $P<0.05$ ).

Prior to randomization for the chronic study, there was no significant difference in LV geometry among GRMD saline- versus P188-treated animals (Table 1 and Figure 7, A and C). After the 8 -week infusion period, a second hemodynamic protocol was performed on the dystrophic dogs. During the course of the 8-week infusion period, GRMD dogs receiving saline developed significant LV dilation $(P<0.05$; Figure 7$)$. Dramatically, GRMD animals

\section{Figure 3}

Baseline hemodynamic function in GRMD animals. (A and B) Representative LV pressure tracings during inferior vena cava occlusion in wild-type (A) and GRMD (B) hearts. (C and D) Summary of enddiastolic volume (C) and stroke volume (D) at rest (black bars) and in response to dobutamine (white bars). Summaries of the change in mean end-diastolic volume (EDV) (E) and end-systolic volume (ESV) $(F)$ observed in response to dobutamine. Values are shown as mean \pm SEM. ${ }^{*} P<0.05$ versus wild-type.

receiving P188 had no alteration in LV geometry, with no progression toward a dilated cardiac phenotype. LV geometry differences between saline and P188-treated GRMD animals were evident throughout the hemodynamic testing protocol (Figure 7C) and were particularly pronounced after the administration of dobutamine (Figure 7, C-F). The dilation of ventricular geometry in the saline GRMD group is consistent with ongoing cardiac injury and necrosis/fibrosis. Hemodynamic data also showed a significant enhancement in the isovolumic relaxation function in P188-treated GRMD compared with saline-treated GRMD animals (Figure 7D). The mechanism by which chronic P188 mediates improvements in diastolic function is not clear, but its determination would likely be of further benefit in DMD patients, where diastolic dysfunction is an early marker of cardiac disease (36).

Myocytes isolated from dystrophic dogs after infusion with P188 or saline showed some significant differences in calcium handling. The amplitude of calcium release and kinetics of relaxation were both improved in myocytes isolated from GRMD dogs receiving chronic P188 infusion (Supplemental Figure 3). These improvements in calcium handling resulted from the improved myocyte health in the group treated with P188, as opposed to a direct action of P188, which is washed out during the myocyte isolation procedure.

To determine the effects of chronic P188 administration on the primary cellular defect of dystrophin-deficient cardiac myocytes, we examined the passive tension-extension properties of isolated myocytes at the end of the infusion protocol (Figure 8A). As seen with myocytes from untreated GRMD dogs (Figure 1), single myocytes isolated from GRMD animals receiving saline were highly sensitive to even small extensions in length. In addition to showing poor myocyte compliance, saline-infused GRMD myocytes were highly susceptible to terminal contracture after mild sarcomeric extension (Figure 8, A and C). Myocytes isolated from GRMD dogs receiving chronic $\mathrm{P} 188$, but with $\mathrm{P} 188$ washed out during the isolation procedure, had
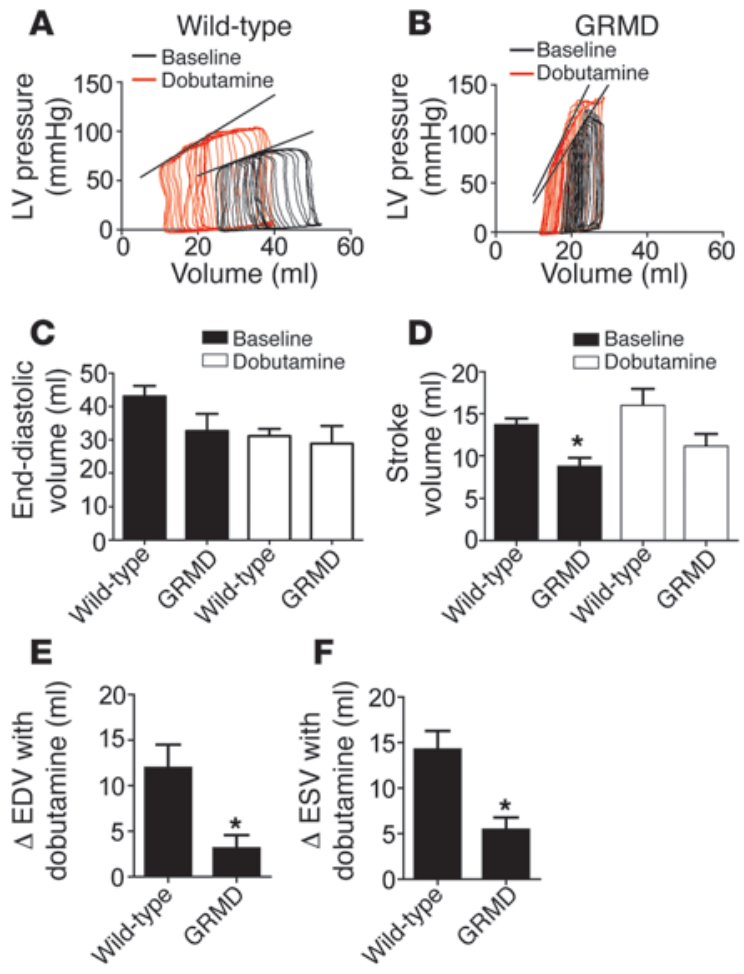


\section{Table 2}

Summary of pretreatment hemodynamic and electrocardiographic parameters at baseline and during acute dobutamine infusion

\begin{tabular}{|c|c|c|c|c|}
\hline & GRMD Baseline & GRMD dobutamine & WT baseline & WT dobutamine \\
\hline Weight (kg) & $13.43 \pm 0.61^{\mathrm{A}}$ & $13.43 \pm 0.61^{A}$ & $18.53 \pm 0.28$ & $18.53 \pm 0.28$ \\
\hline \multicolumn{5}{|l|}{ Systolic function } \\
\hline 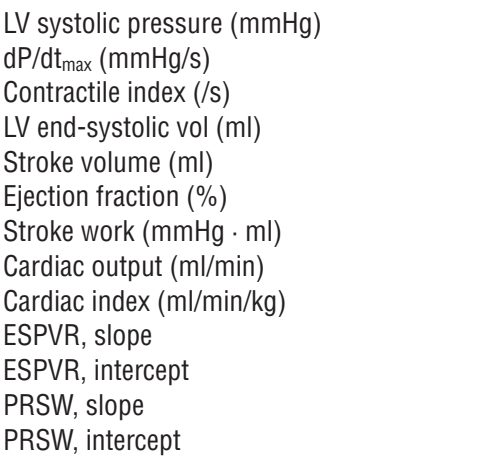 & $\begin{aligned} 110.4 & \pm 5.5^{\mathrm{A}} \\
2,154 & \pm 172 \\
40.7 & \pm 5.3 \\
23.9 & \pm 4.8 \\
8.7 & \pm 1.1^{\mathrm{A}} \\
28.8 & \pm 4.2 \\
748.8 & \pm 103.9 \\
892.9 & \pm 118.3 \\
68.4 & \pm 10.3 \\
4.45 & \pm 0.46^{\mathrm{A}} \\
0.50 & \pm 5.17 \\
45.66 & \pm 5.88 \\
16.01 & \pm 5.56\end{aligned}$ & $\begin{aligned} 131.7 & \pm 3.9 \\
5,756 & \pm 512 \\
77.8 & \pm 6.0 \\
17.8 & \pm 4.6 \\
11.1 & \pm 1.5 \\
42.0 & \pm 4.9 \\
1,215 & \pm 205.6 \\
1,089 & \pm 142.5 \\
84.2 & \pm 14.0 \\
6.51 & \pm 0.83^{A} \\
-1.79 & \pm 3.54 \\
71.62 & \pm 7.30 \\
9.67 & \pm 3.90\end{aligned}$ & $\begin{aligned} 86.91 & \pm 1.9 \\
1,789 & \pm 82 \\
36.9 & \pm 2.0 \\
29.4 & \pm 3.4 \\
13.6 & \pm 0.8 \\
33.4 & \pm 3.5 \\
931.1 & \pm 82.2 \\
1,167 & \pm 84.4 \\
62.8 & \pm 4.1 \\
2.29 & \pm 0.28 \\
-6.91 & \pm 5.80 \\
35.63 & \pm 3.49 \\
17.51 & \pm 4.59\end{aligned}$ & $\begin{aligned} 127.6 & \pm 4.4 \\
6,120 & \pm 528 \\
79.5 & \pm 4.3 \\
15.2 & \pm 2.5 \\
15.9 & \pm 2.1 \\
52.4 & \pm 6.3 \\
1506 & \pm 242.3 \\
1645 & \pm 277.8 \\
89.5 & \pm 15.9 \\
2.89 & \pm 0.57 \\
-5.98 & \pm 8.80 \\
66.21 & \pm 9.55 \\
4.55 & \pm 5.72\end{aligned}$ \\
\hline \multicolumn{5}{|l|}{ Diastolic function } \\
\hline 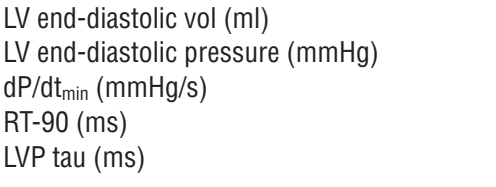 & $\begin{aligned} 32.7 & \pm 5.1 \\
7.05 & \pm 0.38 \\
3.339 & \pm 314 \\
46.3 & \pm 7.1 \\
27.6 & \pm 1.1\end{aligned}$ & $\begin{aligned} 28.9 & \pm 5.3 \\
8.20 & \pm 0.64 \\
4,862 & \pm 329 A \\
37.4 & \pm 6.4 \\
18.9 & \pm 1.1\end{aligned}$ & $\begin{aligned} 43.1 & \pm 3.1 \\
7.03 & \pm 0.82 \\
2,324 & \pm 141 \\
48.8 & \pm 2.4 \\
26.6 & \pm 1.0\end{aligned}$ & $\begin{array}{c}31.1 \pm 2.2 \\
10.5 \pm 1.4 \\
3,654 \pm 245 \\
42.0 \pm 1.6 \\
18.1 \pm 1.7\end{array}$ \\
\hline \multicolumn{5}{|l|}{ Electrocardiograph } \\
\hline $\begin{array}{l}\text { Heart rate (bpm) } \\
\text { P-R interval } \\
\text { Q/R ratio (lead II) }\end{array}$ & $\begin{aligned} 104.9 & \pm 5.4^{\mathrm{A}} \\
94.1 & \pm 3.8^{\mathrm{A}} \\
0.435 & \pm 0.021^{\mathrm{A}}\end{aligned}$ & $\begin{aligned} 100.1 & \pm 6.5 \\
89.4 & \pm 9.9 \\
0.433 & \pm 0.029\end{aligned}$ & $\begin{aligned} 86.0 & \pm 4.7 \\
124.2 & \pm 8.0 \\
0.236 & \pm 0.033\end{aligned}$ & $\begin{aligned} 105.1 & \pm 11.7 \\
94.8 & \pm 3.4 \\
0.224 & \pm 0.037\end{aligned}$ \\
\hline \multicolumn{5}{|l|}{ Arterial function } \\
\hline $\begin{array}{l}\text { Systemic arterial BP, systolic }(\mathrm{mmHg}) \\
\text { Systemic arterial BP, diastolic }(\mathrm{mmHg}) \\
\text { Arterial elastance }(\mathrm{mmHg} / \mathrm{ml}) \\
\text { Total peripheral resistance }(\mathrm{mmHg} \cdot \mathrm{min} / \mathrm{ml})\end{array}$ & $\begin{aligned} 136.9 & \pm 8.6^{A} \\
74.8 & \pm 5.3^{A} \\
16.0 & \pm 2.5^{A} \\
0.109 & \pm 0.019^{A}\end{aligned}$ & $\begin{aligned} 179.2 & \pm 8.2^{\mathrm{A}} \\
78.0 & \pm 3.2^{\mathrm{A}} \\
14.2 & \pm 2.1^{\mathrm{A}} \\
0.132 & \pm 0.025^{\mathrm{A}}\end{aligned}$ & $\begin{aligned} 105.2 & \pm 3.7 \\
59.6 & \pm 2.9 \\
6.3 & \pm 2.3 \\
0.058 & \pm 0.018\end{aligned}$ & $\begin{aligned} 137.8 & \pm 7.0 \\
61.0 & \pm 2.8 \\
6.3 & \pm 1.6 \\
0.064 & \pm 0.021\end{aligned}$ \\
\hline
\end{tabular}

Values are shown as mean \pm SEM $(n=7-10)$. ${ }^{A} P<0.05$ versus wild-type. ESPVR, end-systolic pressure-volume relationship; PRSW, preload recruitable stroke work; RT-90, relaxation time to $90 \%$ of peak height.

passive extension properties similar to those of dystrophic myocytes from untreated animals (Figure 8A), demonstrating that the P188 activity is readily reversed. However, acute administration of P188 to isolated GRMD myocytes, regardless of previous treatment, corrected the passive tension-extension relationship to levels similar to those of dystrophin-replete canine myocytes (Figure 8, B and C). These data demonstrate that the severely attenuated passive extension-tension relationship of GRMD myocytes occurs secondary to the formation of membrane tears that are corrected in the presence of P188.

Utrophin expression in GRMD myocardium. The mechanism underlying blunted cellular compliance of GRMD cardiac myocytes is unknown. The $m d x$ heart shows a marked upregulation of the dystrophin homolog utrophin, which may explain a mechanism by which $m d x$ mice are relatively spared from the severe pathology observed in dystrophin-deficient dogs or humans $(37,38)$. To determine whether similar upregulation occurs in GRMD hearts, we assessed utrophin levels in crude microsomal membranes isolated from the hearts of 2 untreated GRMD and 3 wild-type dogs. These studies revealed that, in contrast to the $m d x$ mouse, the GRMD dogs lack upregulation of utrophin in their hearts (Figure 9). Furthermore, the expression of utrophin was unaltered by the chronic infusion of P188 (Supplemental Figure 4).

\section{Discussion}

This study provides the first evidence to our knowledge of an effective long-term treatment for the pronounced cardiomyopathy in a large animal model of DMD. Chronic intravascular infusion of membrane-sealing tri-block copolymer P188 was found to be safe and highly effective at limiting myocardial fibrosis and preventing the emergence of LV dilation in GRMD dogs, a severely affected animal model of DMD. Because the natural history of muscular dystrophy in canines and humans is comparable, both in severity and timing, and as poloxamers are used in human patients for other indications (39-42), these findings raise the potential of translating this approach as a viable treatment modality for human DMD patients.

Mechanism of cardiac disease severity in GRMD animals. The only published studies on membrane sealants and the cardiomyopathy of DMD have centered on single-dose acute applications of poloxamer in the $m d x$ mouse (30). The $m d x$ mouse model of DMD continues to be valuable in elucidating mechanisms of disease and for the testing of new experimental therapeutics. One challenge for these investigations, however, is the somewhat mild cardiomyopathy in the $m d x$ mouse compared with the severely affected GRMD animals. In addition, intracellular $\mathrm{Ca}^{2+}$ handling in the mouse heart 
A

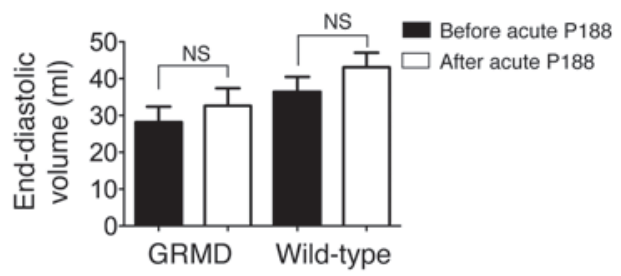

B
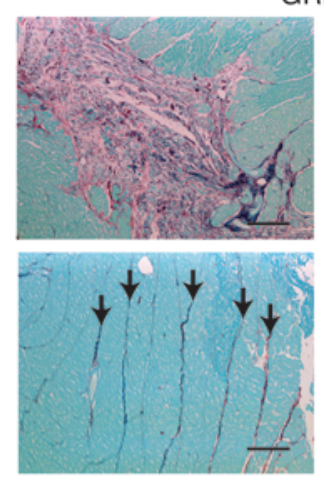

C

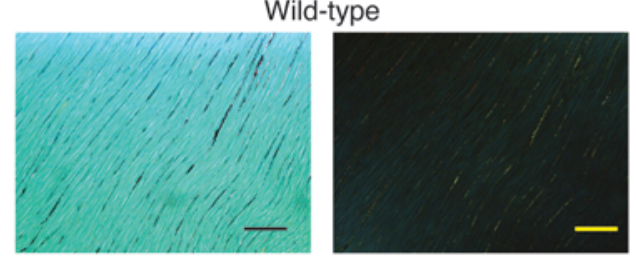

is markedly different from that in the dog heart, which is highly comparable to human myocardium (43). This is an important consideration, as the primary defect in the cardiac myocytes of $m d x$ mice is micro-tears in the sarcolemma during passive cell distension leading to transient $\mathrm{Ca}^{2+}$ entry. This leads to heightened passive tension-extension properties of the $m d x$ myocyte (30). Because GRMD animals are known to have pronounced cardiomyopathy $(25,28,29,44)$, it was important to ascertain passive tensionextension function of the membrane-intact GRMD myocyte for comparison with that obtained recently in the $m d x$ myocyte (30). Results here showed significantly reduced compliance of GRMD compared with $m d x$ myocytes (Figure 1) (30). Even for small excursions in length from baseline sarcomere lengths $(>1.8 \mu \mathrm{m})$, GRMD myocytes had abnormally high passive tension not seen previously in $m d x$ or wild-type cardiac myocytes. The quantitative difference in compliance defects between dystrophic dog and mouse isolated myocytes suggests important mechanistic differences in cell structure/function in these models. Another important new finding of this study is that membrane-intact cardiac myocytes from GRMD

\section{Figure 5}

Summary of disease biomarkers during chronic administration of P188 in GRMD animals. (A) Outline of study design for the chronic administration of P188 $(n=4)$ or saline $(n=4)$ in GRMD animals. (B-E) Serum chemistry data from samples take biweekly throughout the chronic infusion period; shown are (B) CTnl (dashed line indicates wild-type canine values), (C) CK, (D) aspartate transferase (AST) and alkaline phosphatase (ALP), and (E) blood urea nitrogen (BUN) and creatinine. Values are shown as mean \pm SEM; 4-8 animals per group. ${ }^{*} P<0.05$, significant treatment effect by 2-way ANOVA analysis.

\section{Figure 4}

Preexisting fibrotic lesions and ventricular volume in GRMD dogs. (A) Assessment of end-diastolic volume before and after acute infusion of P188 in GRMD and wild-type canines. Values are shown as mean \pm SEM. (B) Representative Sirius red-stained histopathological sections from untreated adult GRMD animals. In brightfield images (left), collagen appears red; in polarized light, Sirius red-stained collagen exhibits birefringence, allowing it to be visualized as green-yellow light (right). Upper images show a large lesion with extensive fibrosis and degenerating myocytes. Lower images show strands of collagen that extend through areas of relatively normal myocardium (arrows). (C) Identical stains of wild-type myocardium. Scale bars: $100 \mu \mathrm{m}$.

dogs lack a key cellular adaptation present in the $m d x$ myocardium in terms of upregulation of the dystrophin homolog utrophin $(37,38)$. Utrophin is highly homologous to dystrophin, sharing many of the same structural domains (45). In wild-type mice, very little utrophin is expressed in the heart. In the $m d x$ mouse, where dystrophin is absent, utrophin protein expression is significantly increased and localized to the sarcolemma, where dystrophin is normally found $(37,38)$. Furthermore, transgenic expression of utrophin in dystrophin-deficient muscle prevents pathology and restores normal function $(46,47)$. Utrophin is thought to function as a dystrophin surrogate, providing increased structural integrity to the dystrophin-deficient membrane, reducing the prevalence of membrane tears, and limiting myocytes loss. In this context, our new finding that utrophin expression is not increased in the GRMD myocardium provides a mechanism to account for the increased mechanical stiffness and susceptibility to damage of the GRMD compared with $m d x$ myocardium.

These dystrophic myocyte structure-function results form the core of a model to help explain the marked organ-level disease severity in GRMD animals (Figure 10). Findings here and elsewhere $(28,30)$ show that myocyte passive distension, as would normally occur during diastolic filling of the ventricle, are shifted markedly leftward in dystrophin deficiency (Figure 10A), indicating marked
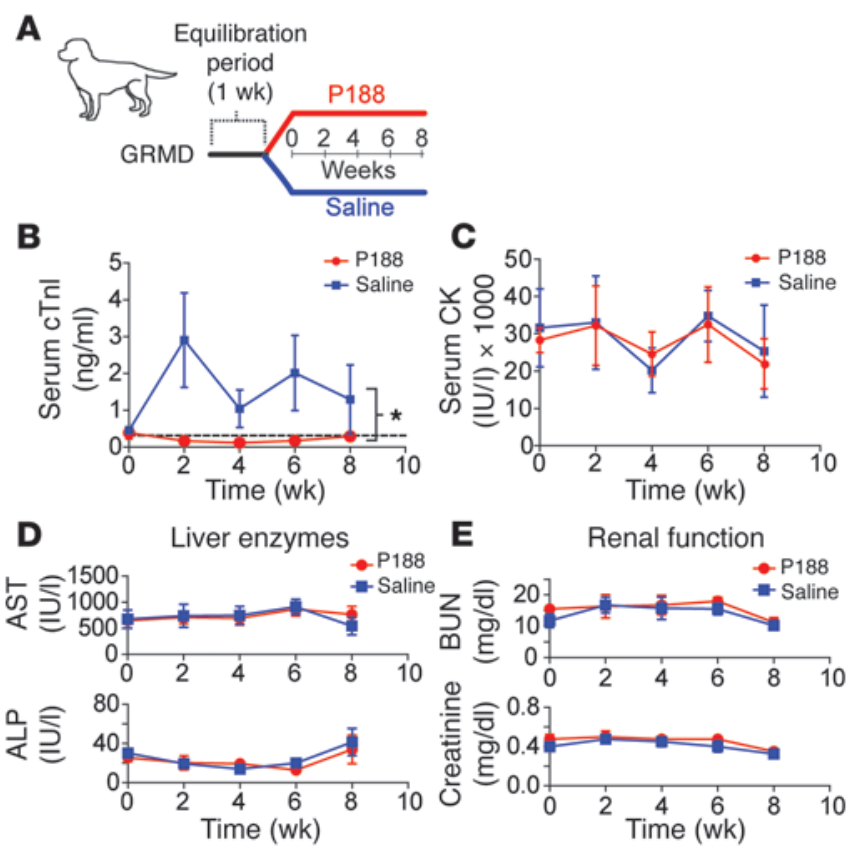


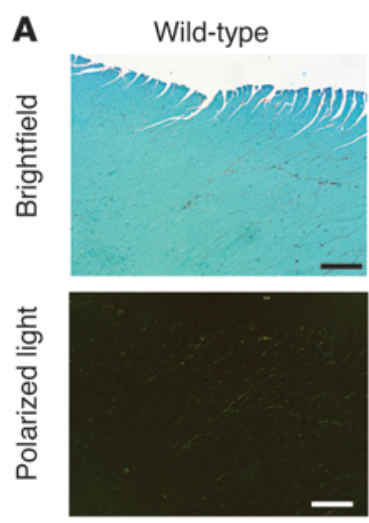

B

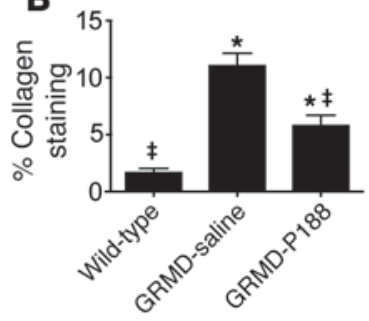

GRMD saline-treated
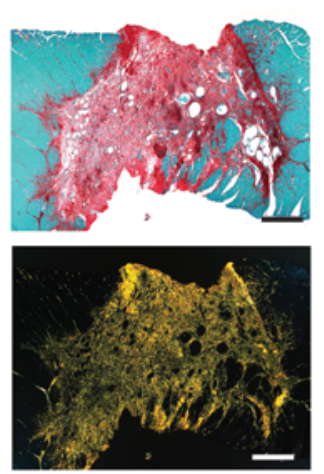

C

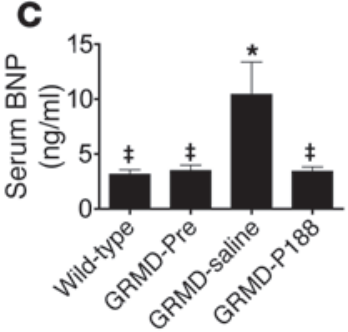

GRMD P188-treated
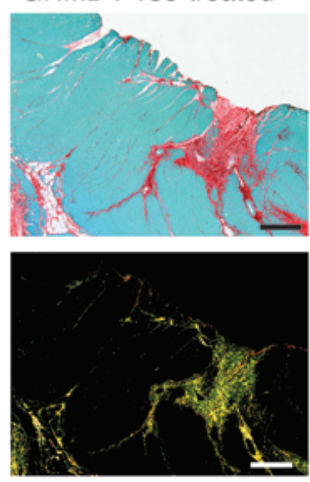

Figure 6

Chronic P188 treatment limits myocardial fibrosis and blocks elevation in BNP in GRMD animals. (A) Sirius red staining of myocardial sections reveals extensive fibrosis in GRMD hearts. Collagen appears red in brightfield images and yellow-green in polarized images. Scale bars: $400 \mu \mathrm{m}$. (B) Quantification of collagen content from 16-24 sections from 4 dogs in each group. (C) Serum BNP levels taken before (GRMD-Pre) and after the chronic infusion protocol. Values are shown as mean \pm SEM; 4-8 animals per group. ${ }^{*} P<0.05$ versus wild-type; $\ddagger P<0.05$ versus GRMD (saline) group. All data were analyzed with 1-way ANOVA and a Bonferroni's multiple comparisons post-hoc test. stiffness of the membrane-intact cell. In model systems whereby dystrophin deficiency is accompanied by upregulation of utrophin (e.g., $m d x$ ), myocyte compliance is partially restored toward normal (Figure 1B and Figure 10B). Differences in the severity of the cell compliance defect could, in turn, account for the greater fibrosis in GRMD than $m d x$ hearts owing to increased myocyte loss. The results obtained here suggest that lack of upregulation of utrophin in GRMD myocytes causes significant membrane destabilization, limiting cell membrane integrity and therefore compliance, culminating in worsening organ-level pathology (Figure 10B).

Chronic membrane sealant and the dystrophic heart. The presence of membrane micro-tears constitutes a primary cellular deficit of dystrophin-deficient cardiac myocytes that is readily corrected by the direct application of membrane sealants. Membrane sealants prevent breaches in membrane barrier function by inserting into the disrupted phospholipid bilayers of membranes $(30,48)$. In this context, it is surprising that P188 had no acute effect on in vivo hemodynamics in GRMD animals. This contrasts with P188's immediate acute effects of restoring normal ventricular geometry to $m d x$ hearts in vivo (30). We speculate that lack of an acute organ-level effect in GRMD animals relates to the greater fibrosis present in the adult GRMD heart, such that it provides temporary structural support, preventing the acute expansion of the ventricle despite the normalization of cardiac myocyte passive properties. However, as cell lysis and subsequent fibrotic remodeling ensues, GRMD progresses to a dilated cardiomyopathy, as reported here (Figure $8 \mathrm{~B}$ ).

What accounts for the beneficial effects of long-term application of P188 on GRMD hearts? We propose that long-term administration of P188 maintains myocardial membrane function and prevents myocardial necrosis, as shown by significantly lower levels of $\mathrm{cTnI}$ in the treated GRMD dogs. The prevention of myocardial necrosis by chronic P188 can also account for the reduction in the amount of fibrosis in the P188-treated GRMD heart. Attenuation of the number and size of lesions present in the heart would decrease the stress on the remaining viable myocardium and serve to limit cardiac remodeling and block ventricular dilation. It is of note that the progression of disease appears to accelerate after the initiation of the chronic infusion protocol in GRMD dogs receiving saline alone, as measured by biomarkers of cardiac necrosis. The reasons for this observed acceleration are likely to be multifactorial, including residual cardiac injury after initial surgical procedure, increased manipulation during the infusion proto$\mathrm{col}$, and the extra weight of the infusion pump and solutions on these animals. However, none of these would be expected to disproportionately affect saline-infused animals. All of these factors would increase the stress on viable cardiac myocytes. This extra stress would be expected to increase the frequency of mechanically induced disruptions of membrane integrity that are an important inciting event in the pathophysiology of dystrophin deficiency in the heart. This accelerated disease progression makes the protective effects of P188 even more notable. Under conditions in which untreated dystrophic hearts are deteriorating, P188 appears to halt the progression of the disease.

The loss of membrane integrity results from mechanical sheer stresses placed on the membrane by the movements of the extracellular matrix and the underlying myofilaments. These stresses are more frequent and greater in magnitude during times of cardiac stress, suggesting that membrane sealants may be particularly effective during these events. One important and predictable cardiac stressor is general anesthesia. DMD patients commonly undergo major surgical procedures to correct orthopedic dysfunction. These procedures have the potential to result in significant cardiac stress secondary to blood loss and hypotension. Moreover, in untreated GRMD animals, general anesthesia alone was sufficient to induce significant increases in cTnI, a marker of myocardial necrosis and cardiac injury. Given the low rate of cardiac regeneration, the progressive and unabated loss of cardiac myocytes would ultimately cause ventricular dilation and deficits in global cardiac function. Thus, cardiac myocyte membrane damage is an inciting event that initiates a cascade of deleterious processes that eventually lead to cardiomyopathy and ventricular 

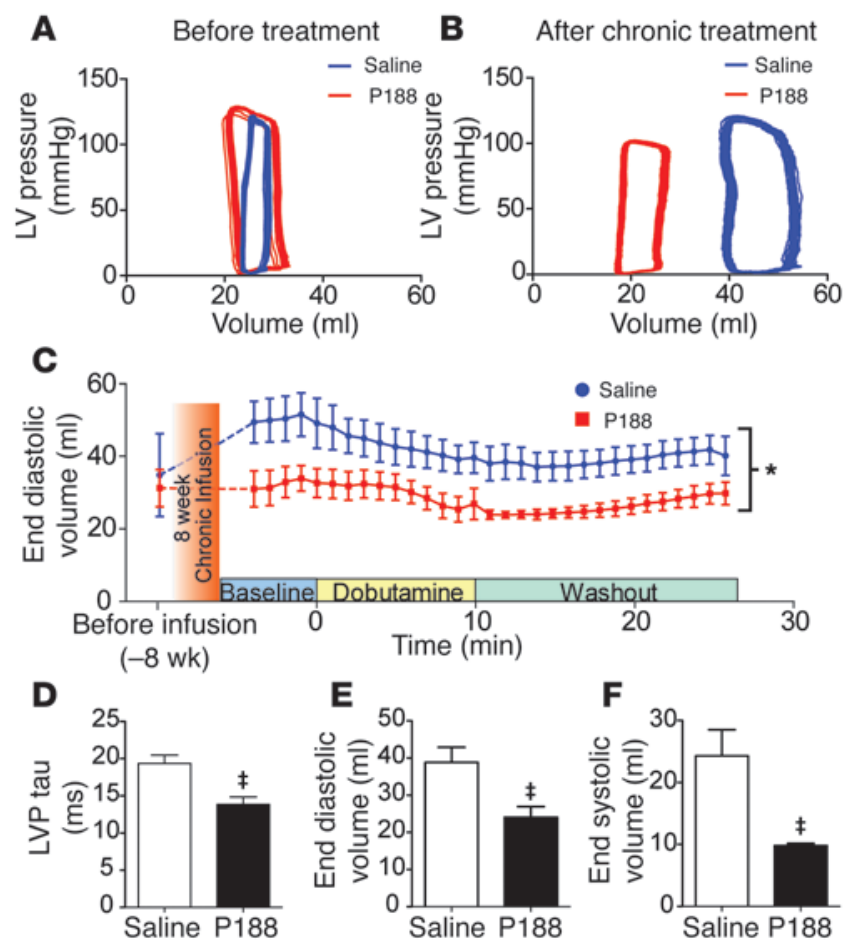

remodeling. Our results suggest that application of membrane sealants during times of anticipated cardiac stress would disrupt this spiral of cardiac damage early on, thus halting the progression of the dystrophic heart disease.

In summary, our data establish a cellular basis for the severe cardiac damage observed in dystrophin-deficient dogs. The cellular defect of marked reduction in myocyte compliance, due to dystrophin deficiency and the absence of utrophin upregulation, was corrected by membrane sealant P188. Chronic application of P188 was highly effective in preventing cardiac injury and ventricular remodeling in a severely affected large animal model of DMD. P188 is FDA approved for short-term applications in humans. In this chronic study in GRMD animals, P188 was also shown to be safe in GRMD animals. These findings have significant clinical relevance, as P188 has the potential to halt the progression of dystrophic cardiomyopathy, which is the second most common cause of death in DMD patients. In addition, we further speculate that membrane sealant therapy may extend to a myriad of acquired cardiac diseases in which dystrophin struc-

\section{Figure 8}

In vitro passive tension-extension relationships in intact GRMD cardiac myocytes after chronic infusion. After the 8-week infusion, myocytes were isolated from P188- (red) and saline-infused (blue) GRMD and wild-type dogs. Passive tension-extension relationships of dystrophic cardiomyocytes in the absence $(\mathbf{A})$ and presence $(\mathbf{B})$ of acute application of $150 \mu \mathrm{M}$ P188 are shown. The black curve in B is derived from wild-type myocyte data in $\mathbf{A}$ and is included as a reference. SL, sarcomere length. (C) Comparisons of maximum stable sarcomere length (Max SL) between the groups. ${ }^{*} P<0.05$ versus wild-type; t $P<0.05$ versus chronic saline GRMD group; $¥ P<0.05$ versus chronic P188 GRMD group. Values are shown as mean \pm SEM. All points were derived from 5-7 myocytes isolated from 4 -5 dogs. All data were analyzed with 1-way ANOVA and Bonferroni's multiple comparisons post-hoc test. MD, GRMD.

\section{Figure 7}

Eight weeks of P188 administration prevents LV remodeling in GRMD animals. Catheter-based intraventricular recordings demonstrate significant ventricular remodeling in GRMD dogs receiving saline infusion. (A and $\mathbf{B}$ ) Representative tracings of pressure-volume loops from before the treatment (A) and after the chronic infusion protocol (B). (C) Monitoring of LV end-diastolic volume during the terminal hemodynamic proto$\mathrm{col}$. Offset at the far left are baseline data from the initial hemodynamic protocol prior to chronic infusion. ${ }^{*} P<0.05$ (see Methods). (D-F) Comparisons of hemodynamic data taken during the peak of the dobutamine response ( $\approx 10$ minutes after infusion); LV pressure tau (D), end-diastolic volume (E), and end-systolic volume (F). Groups consist of 3-4 dogs. $\ddagger P<0.05$ by $t$ test. For $\mathbf{C}-\mathbf{F}$, values are shown as mean \pm SEM.

ture-function and membrane integrity are known to be compromised, including viral myocarditis, myocardial ischemia, and heart failure (49-52).

\section{Methods}

Animals. Adult GRMD dogs were obtained from a colony maintained at the University of North Carolina, Chapel Hill. GRMD dogs were identified based on elevation of serum CK and subsequently developed characteristic clinical signs. Genotype was confirmed by PCR in most dogs. Approximately age-matched, heartworm-negative normal dogs of mixed breed provided by R \& R Research were used as wild-type dogs. All animals were used and cared for according to principles outlined in the NIH Guide for the care and use of laboratory animals (NIH publication no. 85-23. Revised 1985) and protocols reviewed and approved by the Institutional Animal Care and Use Committee of the University of Minnesota.

Vascular access port placement and hemodynamics. Premedication consisted of morphine ( $1 \mathrm{mg} / \mathrm{kg}$; i.m.) and midazolam ( $0.3 \mathrm{mg} / \mathrm{kg}$; i.m.); some of the wildtype dogs required a supplemental dose of acepromazine $(0.05 \mathrm{mg} / \mathrm{kg}$; i.m.). Anesthesia was induced with intravenous propofol $(4 \mathrm{mg} / \mathrm{kg})$ and maintained at a surgical plane of anesthesia with either isoflurane or sevoflurane. After placement of a femoral arterial catheter, the left jugular vein and carotid artery were isolated. A 6 French introducer was inserted into the left carotid and secured with a purse-string suture. Under fluoroscopic guidance, a 5 French 10 electrode pressure-volume catheter (SPR-554-11, Millar Instruments) was advanced through the aortic valve into the lumen of the left ventricle and positioned to give strong signals in all 7 segments and appropriate
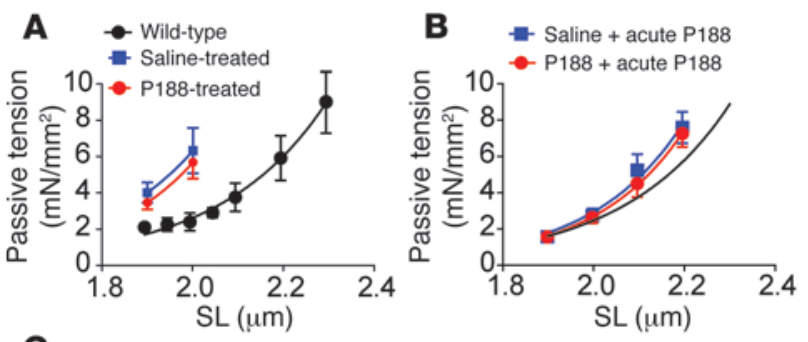

C

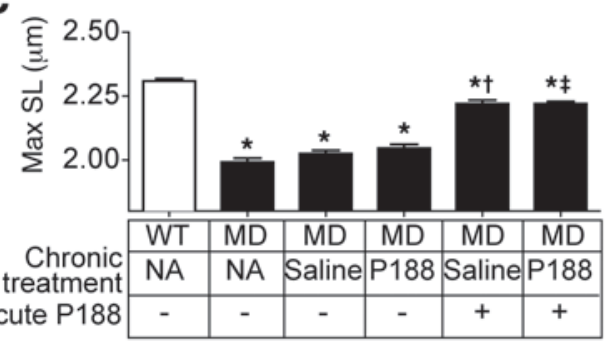


A

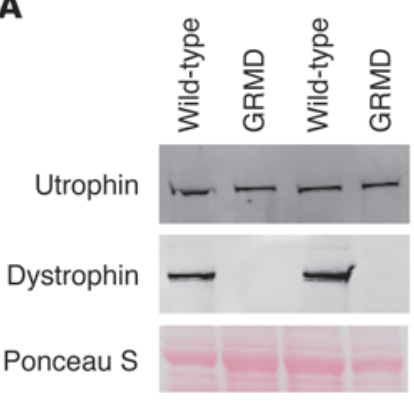

B

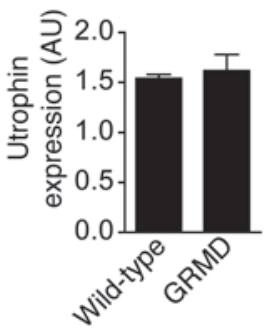

pressure-volume loops. The catheter interfaced with a MPVS Ultra signaling conditioning unit (Millar Instruments), and data were collected by a DAQ-16 acquisition unit and Ponemah software package (DSI). During the initial hemodynamic protocol, measurements were taken at baseline, after an infusion of dobutamine $(15 \mu \mathrm{g} / \mathrm{kg} / \mathrm{min})$, after a return to baseline values, after an infusion of P188 $(460 \mathrm{mg} / \mathrm{kg})$, and after a second dobutamine infusion. Concurrently with the hemodynamic study, a pulmonary artery catheter was placed, its position verified by observation of a pulmonary artery pres-

\section{Figure 9}

Utrophin expression in GRMD hearts. (A) Western blot analysis of utrophin and dystrophin $\mathrm{KCl}$-washed crude microsomal membranes isolated from wild-type and GRMD dog hearts. (B) Quantification of the levels of utrophin expression observed in $\mathbf{A}$.

sure tracing. Also implanted was a stainless steel vascular access port (VAP; CP4AC-5IS-SS, Access Technologies). The VAP was implanted approximately $5 \mathrm{~cm}$ caudal to the spine of the scapula and approximately $10 \mathrm{~cm}$ from the dorsal midline. A 22-gauge right-angle Huber needle was introduced into the VAP and secured with 2-0 Prolene. A 5 French silicone catheter was tunneled subcutaneously to the neck incision. After completion of the hemodynamic protocol, a bolus of $10 \% \mathrm{NaCl}$ was injected directly into the pulmonary circulation, allowing for calibration of the conductance volume. After removal of the pulmonary artery catheter, the heparin-flushed catheter connected to the VAP was then introduced into the jugular vein such that the tip was near the right atrium. At the completion of the hemodynamic protocol, the carotid was repaired, all skin incisions were closed, and the animals were allowed to recover. Average induction to extubation period was approximately 6 hours. Once fully recovered, animals were placed in fitted jackets (Lomir Biomedical) that protected the VAP site.

A

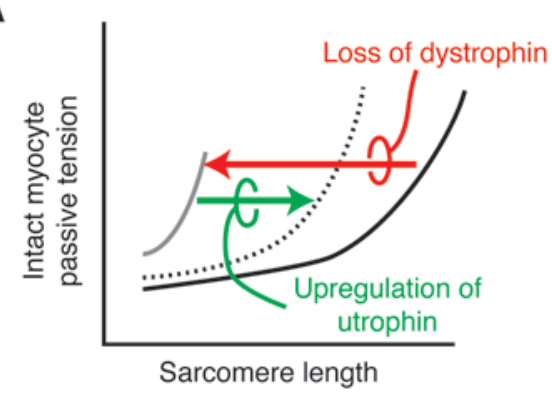

B Wild-type $m d x$ GRMD

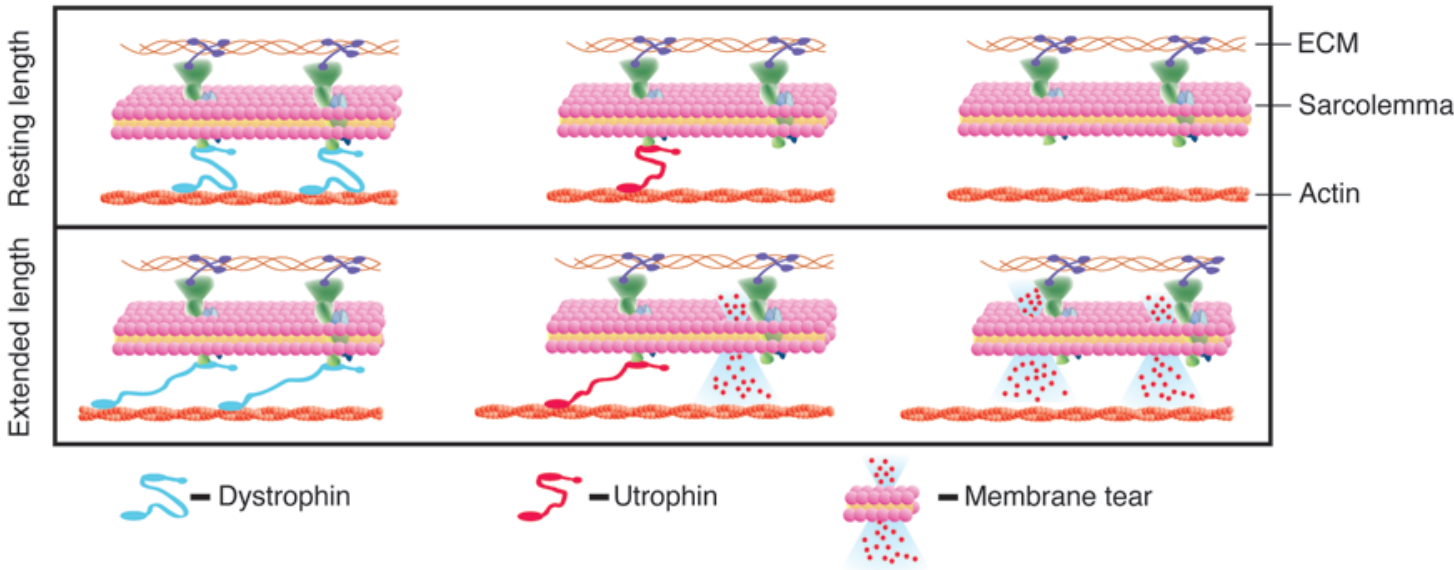

\section{Figure 10}

Model of the cellular basis for severe cardiomyopathy in GRMD animals. (A) A schematic of membane-intact cardiac myocyte passive tensionextension curves. In the presence of dystrophin, myocytes show compliance throughout the working sarcomere length range (black line). In the absence of dystrophin, the cardiac myocytes become very noncompliant and resistant to passive extensions (gray line), as seen in GRMD myocytes. Upregulation of utrophin partially restores the passive extension-tension properties of the dystrophin-deficient myocytes (dotted line), as seen in the $m d x$ myocytes. (B) Cellular model depicting the normal mechanical stabilizing function of dystrophin (blue) in wild-type animals. In wild-type cardiac myocytes, upon passive cell extension (lower scheme), dystrophin protects the membrane from damage. In the $m d x$ myocytes, dystrophin is absent, but utrophin (red) is upregulated and partially replaces the mechanical buffering properties of dystrophin. This provides some, but not full protection of the membrane from mechanical damage. Hence, with cell extension, there is some membrane damage. In the dystrophin-deficient dog myocytes (GRMD), utrophin is not upregulated. Thus, upon cell extension, there is more damage to the membrane. 
Chronic infusion protocol. An ambulatory infusion pump (Ambit, Sorenson Medical) was inserted into a jacket pocket, and a 500-ml i.v. bag was placed into a second pocket. In total, the jacket, pump, and a full bag of fluid weighed slightly more than $1 \mathrm{~kg}$ ( $6 \%-9 \%$ of body weight). Saline infusions began the morning after the implantation of the VAP and were continued for 7 days, at which time baseline blood samples were taken and dogs in the P188 treatment group started receiving P188 infusion. National Formulary grade $\mathrm{P} 188$ was provided by BASF.

Myocyte isolation. After the terminal hemodynamic study, the animals were euthanized by a barbiturate overdose, the chest was rapidly opened through a lateral thoracotomy, and the heart was removed and placed into ice-cold Krebs solution. Sections of myocardium were cut into 5 -mm cubes and digested in a solution containing nominal calcium, type 2 collagenase (250-300 U/ml; Wothington Biochemical Corp.), and hyaluronidase (250-500 U/ml; H3506, Sigma-Aldrich). After an initial period of digestion, the heart sections were gently triturated to further disperse the isolated cells. Calcium was reintroduced in a stepwise manner; calcium-tolerant myocytes were then utilized. Other representative portions of myocardium were placed in formalin and embedded in paraffin for sectioning.

Image acquisition and analysis. Images of sectioned tissues were obtained on an inverted Axio Observer Z-1 with a motorized stage (Zeiss). Montage images were collected and stitched by AxioVision software (Zeiss). Composite images were analyzed by a custom-written edge-detection routine using MatLab (R2008a, MathWorks).

Western blotting. KCl-washed crude microsomes were prepared as previously described $(24,53)$. Membrane proteins $(20 \mu \mathrm{g})$ were separated by SDS-PAGE using a $4 \%-20 \%$ gradient (Bio-Rad). Proteins were then transferred to nitrocellulose by the procedures of Towbin et al. (54). Transferred proteins were detected using 1:1,000 polyclonal anti-dystrophin (Abcam) or monoclonal anti-utrophin (MANCHO3; DSHB). The MANCHO3 developed by G.E. Morris (55) was obtained from the Developmental Studies Hybridoma Bank developed under the auspices of the National Institute of Child Health and Human Development and maintained by the University of Iowa Department of Biology. Band intensities were quantified by an Odyssey scanner (LiCor), and secondary antibodies were goat anti-mouse IgG conjugated to either Alexa Fluor 680 (Invitrogen) or IRDye 800 (Rockland Immunochemicals).

Statistics. Statistical analysis was performed using Prism version 5.0 (GraphPad Sofware), and a custom SAS-PROC MIXED model of covariance accounting for both fixed and random effects was used to perform analysis of repeated measures (SAS). Statistical significance was defined as $P<0.05$. Data are presented as mean \pm SEM.

\section{Acknowledgments}

This study was supported by funds from the NIH and the Foundation to Eradicate Duchenne to J.M. Metzger and from the MDA to D. Townsend. We thank Eric Devaney for assistance in developing the surgical procedures; John Connett for statistical assistance; Guadalupe Guerrero-Serna for technical assistance; Janet and Dan Bogan for care and transport of GRMD dogs; and the Lillehei Heart Institute, University of Minnesota, for support.

Received for publication October 2, 2009, and accepted in revised form January 27, 2010.

Address correspondence to: DeWayne Townsend, 6-125 Jackson Hall, 321 Church Street SE, Minneapolis, MN 55455. Phone: 612.625.6873; Fax: 612.625.5149; E-mail: town0045@umn.edu.

Jennifer Davis's present address is: Molecular Cardiology, Children's Hospital Cincinnati, Ohio.

Michael Shillingford's present address is: Department of Surgery, University of Florida, Gainesville.

Immanuel Turner's present address is: Department of Surgery, Duke University, Durham, North Carolina.
1. Emery AEH, Muntoni F. Clinical features. In: Emery AEH, ed. Duchenne Muscular Dystrophy. Oxford, United Kingdom: Oxford University Press; 2003:26-45.

2. Hoffman EP, Brown RH Jr, Kunkel LM. Dystrophin: the protein product of the Duchenne muscular dystrophy locus. Cell. 1987;51(6):919-928.

3. Eagle M, et al. Managing Duchenne muscular dystrophy - the additive effect of spinal surgery and home nocturnal ventilation in improving survival. Neuromuscul Disord. 2007;17(6):470-475.

4. Finsterer J, Stollberger C. The heart in human dystrophinopathies. Cardiology. 2003;99(1):1-19.

5. Moriuchi T, Kagawa N, Mukoyama M, Hizawa K. Autopsy analyses of the muscular dystrophies. Tokushima J Exp Med. 1993;40(1-2):83-93.

6. Bogdanovich S, et al. Functional improvement of dystrophic muscle by myostatin blockade. Nature. 2002;420(6914):418-421.

7. Fletcher S, et al. Morpholino oligomer-mediated exon skipping averts the onset of dystrophic pathology in the mdx mouse. Mol Ther. 2007;15(9):1587-1592.

8 . Gregorevic P, et al. Systemic delivery of genes to striated muscles using adeno-associated viral vectors. Nat Med. 2004;10(8):828-834.

9. Sampaolesi M, et al. Mesoangioblast stem cells ameliorate muscle function in dystrophic dogs. Nature. 2006;444(7119):574-579.

10. Wang Z, et al. Sustained AAV-mediated dystrophin expression in a canine model of Duchenne muscular dystrophy with a brief course of immunosuppression. Mol Ther. 2007;15(6):1160-1166.

11. Welch EM, et al. PTC124 targets genetic disorders caused by nonsense mutations. Nature.
2007;447(7140):87-91

12. Wu B, etal. Effective rescue of dystrophin improves cardiac function in dystrophin-deficient mice by a modified morpholino oligomer. Proc Natl Acad Sci U S A. 2008;105(39):14814-14819.

13. Cerletti $M$, et al. Dystrophic phenotype of canine $\mathrm{X}$-linked muscular dystrophy is mitigated by adenovirus-mediated utrophin gene transfer. Gene Ther. 2003;10(9):750-757.

14. Chetboul V, et al. Diagnostic potential of natriuretic peptides in the occult phase of golden retriever muscular dystrophy cardiomyopathy. J Vet Intern Med. 2004;18(6):845-850.

15. Dell'Agnola C, et al. Hematopoietic stem cell transplantation does not restore dystrophin expression in Duchenne muscular dystrophy dogs. Blood. 2004;104(13):4311-4318.

16. McClorey G, Moulton HM, Iversen PL, Fletcher $\mathrm{S}$, Wilton SD. Antisense oligonucleotide-induced exon skipping restores dystrophin expression in vitro in a canine model of DMD. Gene Ther. 2006;13(19):1373-1381.

17. Ohshima $S$, et al. Transduction efficiency and immune response associated with the administration of AAV8 vector into dog skeletal muscle. $\mathrm{Mol}$ Ther. 2009;17(1):73-80.

18. Parker MH, Kuhr C, Tapscott SJ, Storb R. Hematopoietic cell transplantation provides an immunetolerant platform for myoblast transplantation in dystrophic dogs. Mol Ther. 2008;16(7):1340-1346.

19. Qiao C, et al. Hydrodynamic limb vein injection of AAV8 canine myostatin propeptide gene in normal dogs enhances muscle growth. Hum Gene Ther 2009;20(1):1-10.
20. Wang $Z$, et al. Immunity to adeno-associated virusmediated gene transfer in a random-bred canine model of Duchenne muscular dystrophy. Hum Gene Ther. 2007;18(1):18-26.

21. Yokota T, et al. Efficacy of systemic morpholino exon-skipping in Duchenne dystrophy dogs. Ann Neurol. 2009;65(6):667-676.

22. Yuasa $\mathrm{K}$, et al. Injection of a recombinant AAV serotype 2 into canine skeletal muscles evokes strong immune responses against transgene products. Gene Ther. 2007;14(17):1249-1260.

23. Cooper BJ, et al. The homologue of the Duchenne locus is defective in X-linked muscular dystrophy of dogs. Nature. 1988;334(6178):154-156.

24. Townsend D, et al. Systemic administration of micro-dystrophin restores cardiac geometry and prevents dobutamine-induced cardiac pump failure. Mol Ther. 2007;15(6):1086-1092.

25. Valentine BA, Cummings JF, Cooper BJ. Development of Duchenne-type cardiomyopathy. Morphologic studies in a canine model. Am J Pathol. 1989;135(4):671-678.

26. Kornegay JN, Tuler SM, Miller DM, Levesque DC. Muscular dystrophy in a litter of golden retriever dogs. Muscle Nerve. 1988;11(10):1056-1064.

27. Sharp NJ, et al. An error in dystrophin mRNA processing in golden retriever muscular dystrophy, an animal homologue of Duchenne muscular dystrophy. Genomics. 1992;13(1):115-121.

28. Moise NS, et al. Duchenne's cardiomyopathy in a canine model: electrocardiographic and echocardiographic studies. J Am Coll Cardiol. 1991;17(3):812-820.

29. Devaux JY, Cabane L, Esler M, Flaouters H, Duboc D. 
Non-invasive evaluation of the cardiac function in golden retriever dogs by radionuclide angiography. Neuromuscul Disord. 1993;3(5-6):429-432.

30. Yasuda S, Townsend D, Michele DE, Favre EG Day SM, Metzger JM. Dystrophic heart failure blocked by membrane sealant poloxamer. Nature. 2005;436(7053):1025-1029.

31. Rodriguez EK, et al. A method to reconstruct myocardial sarcomere lengths and orientations at transmural sites in beating canine hearts. $A m J$ Physiol. 1992;263(1 pt 2):H293-H306.

32. Brutsaert DL, Sys SU. Relaxation and diastole of the heart. Physiol Rev. 1989;69(4):1228-1315.

33. Grindel JM, Jaworski T, Emanuele RM, Culbreth P. Pharmacokinetics of a novel surface-active agent, purified poloxamer 188, in rat, rabbit, dog and man. Biopharm Drug Dispos. 2002;23(3):87-103.

34. Ishikawa K. Cardiac involvement in progressive muscular dystrophy of the Duchenne type. Jpn Heart J. 1997;38(2):163-180.

35. Puchalski MD, et al. Late gadolinium enhancement: precursor to cardiomyopathy in Duchenne muscular dystrophy? Int $J$ Cardiovasc Imaging. 2009;25(1):57-63.

36. Markham LW, et al. Abnormalities of diastolic function precede dilated cardiomyopathy associated with Duchenne muscular dystrophy. J Am Soc Echocardiogr. 2006;19(7):865-871.

37. Matsumura K, Ervasti JM, Ohlendieck K, Kahl SD, Campbell KP. Association of dystrophin-related protein with dystrophin-associated proteins in $\mathrm{mdx}$ mouse muscle. Nature. 1992;360(6404):588-591.

38. Weir AP, Burton EA, Harrod G, Davies KE. A- and B-utrophin have different expression patterns and are differentially up-regulated in $\mathrm{mdx}$ muscle. J Biol Chem. 2002;277(47):45285-45290.

39. Jewell RC, Khor SP, Kisor DF, LaCroix KA, Wargin WA. Pharmacokinetics of RheothRx injection in healthy male volunteers. J Pharm Sci. 1997;86(7):808-812.

40. CORE-Investigators. Effects of RheothRx on mortality, morbidity, left ventricular function, and infarct size in patients with acute myocardial infarction. Collaborative Organization for RheothRx Evaluation (CORE). Circulation. 1997;96(1):192-201.

41. Ballas S. Purified poloxamer 188 for sickle cell vaso-occlusive crisis. Ann Pharmacother. 2004;38(2):320-324.

42. Adams-Graves P, et al. RheothRx (poloxamer 188) injection for the acute painful episode of sickle cell disease: a pilot study. Blood. 1997;90(5):2041-2046.

43. Bers DM. Cardiac excitation-contraction coupling. Nature. 2002;415(6868):198-205.

44. Chetboul V, et al. Tissue Doppler imaging detects early asymptomatic myocardial abnormalities in a dog model of Duchenne's cardiomyopathy. Eur Heart J. 2004;25(21):1934-1939.

45. Tinsley JM, et al. Primary structure of dystrophinrelated protein. Nature. 1992;360(6404):591-593.

46. Tinsley J, et al. Expression of full-length utrophin prevents muscular dystrophy in mdx mice. Nat Med. 1998;4(12):1441-1444.

47. Tinsley JM, Potter AC, Phelps SR, Fisher R, Trickett JI, Davies KE. Amelioration of the dystrophic phenotype of $\mathrm{mdx}$ mice using a truncated utrophin transgene. Nature. 1996;384(6607):349-353.

48. Lee RC, River LP, Pan FS, Ji L, Wollmann RL. Surfactant-induced sealing of electropermeabilized skeletal muscle membranes in vivo. Proc Natl Acad Sci U S A. 1992;89(10):4524-4528.

49. Vatta $M$, et al. Molecular normalization of dystrophin in the failing left and right ventricle of patients treated with either pulsatile or continuous flow-type ventricular assist devices. J Am Coll Cardiol. 2004;43(5):811-817.

50. Vatta M, et al. Molecular remodelling of dystrophin in patients with end-stage cardiomyopathies and reversal in patients on assistance-device therapy. Lancet. 2002;359(9310):936-941.

51. Badorff C, et al. Enteroviral protease 2A cleaves dystrophin: evidence of cytoskeletal disruption in an acquired cardiomyopathy. Nat Med. 1999;5(3):320-326.

52. Lee GH, Badorff C, Knowlton KU. Dissociation of sarcoglycans and the dystrophin carboxyl terminus from the sarcolemma in enteroviral cardiomyopathy. Circ Res. 2000;87(6):489-495.

53. Ohlendieck K, Campbell KP. Dystrophin-associated proteins are greatly reduced in skeletal muscle from mdx mice. JCell Biol. 1991;115(6):1685-1694.

54. Towbin H, Staehelin T, Gordon J. Electrophoretic transfer of proteins from polyacrylamide gels to nitrocellulose sheets: procedure and some applications. Proc Natl Acad Sci U S A. 1979; 76(9):4350-4354.

55. Nguyen TM, et al. Localization of the DMDL geneencoded dystrophin-related protein using a panel of nineteen monoclonal antibodies: presence at neuromuscular junctions, in the sarcolemma of dystrophic skeletal muscle, in vascular and other smooth muscles, and in proliferating brain cell lines. J Cell Biol. 1991;115(6):1695-1700. 